BNL-113909-2017-JA

\title{
Fringing in MonoCam Y4 filter images
}

\author{
J. Brooks, M. Fisher-Levine, and A. Nomerotski
}

Submitted to Journal of Instrumentation

May 2017

Physics Department

Brookhaven National Laboratory

\author{
U.S. Department of Energy \\ USDOE Office of Science (SC), \\ High Energy Physics (HEP) (SC-25)
}

\footnotetext{
Notice: This manuscript has been authored by employees of Brookhaven Science Associates, LLC under Contract No. DE-SC0012704 with the U.S. Department of Energy. The publisher by accepting the manuscript for publication acknowledges that the United States Government retains a non-exclusive, paid-up, irrevocable, world-wide license to publish or reproduce the published form of this manuscript, or allow others to do so, for United States Government purposes.
} 


\section{DISCLAIMER}

This report was prepared as an account of work sponsored by an agency of the United States Government. Neither the United States Government nor any agency thereof, nor any of their employees, nor any of their contractors, subcontractors, or their employees, makes any warranty, express or implied, or assumes any legal liability or responsibility for the accuracy, completeness, or any third party's use or the results of such use of any information, apparatus, product, or process disclosed, or represents that its use would not infringe privately owned rights. Reference herein to any specific commercial product, process, or service by trade name, trademark, manufacturer, or otherwise, does not necessarily constitute or imply its endorsement, recommendation, or favoring by the United States Government or any agency thereof or its contractors or subcontractors. The views and opinions of authors expressed herein do not necessarily state or reflect those of the United States Government or any agency thereof. 


\title{
Fringing in MonoCam Y4 filter images
}

\author{
J. Brooks, ${ }^{a, 1}$ M. Fisher-Levine ${ }^{b}$ and A. Nomerotski ${ }^{a}$ \\ ${ }^{a}$ Brookhaven National Laboratory, \\ Upton, New York 11973, U.S.A. \\ ${ }^{b}$ Department of Astrophysical Sciences, \\ Peyton Hall, Princeton, New Jersey 08544, U.S.A. \\ E-mail: jasonbrooksw@gmail.com
}

\begin{abstract}
We study the fringing patterns observed in MonoCam, a camera with a single Large Synoptic Survey Telescope (LSST) CCD sensor. Images were taken at the U.S. Naval Observatory in Flagstaff, Arizona (NOFS) employing its $1.3 \mathrm{~m}$ telescope and an LSST y4 filter. Fringing occurs due to the reflection of infrared light (700 nm or larger) from the bottom surface of the CCD which constructively or destructively interferes with the incident light to produce a net "fringe" pattern which is superimposed on all images taken. Emission lines from the atmosphere, dominated by hydroxyl $(\mathrm{OH})$ spectra, can change in their relative intensities as the night goes on, producing different fringe patterns in the images taken. We found through several methods that the general shape of the fringe patterns remained constant, though with slight changes in the amplitude and phase of the fringes. We also found that a superposition of fringes from two monochromatic lines taken in the lab offered a reasonable description of the sky data.
\end{abstract}

Keywords: Analysis and statistical methods; Photon detectors for UV, visible and IR photons (solid-state) (PIN diodes, APDs, Si-PMTs, G-APDs, CCDs, EBCCDs, EMCCDs etc)

\footnotetext{
${ }^{1}$ Corresponding author.
} 


\section{Contents}

1 Introduction 1

$2 \mathrm{OH}$ airglow and fringing $\quad 2$

3 MonoCam 3

3.1 Telescope $\quad 4$

3.2 Data acquisition $\quad 4$

3.3 Data reduction $\quad 4$

$\begin{array}{lll}3.4 & \text { Observed patterns } & 4\end{array}$

4 Data Analysis 5

4.1 Subtraction by midnight pattern $\quad 5$

4.2 Principal Component Analysis $\quad 5$

$\begin{array}{lll}4.3 & \text { Slice plot PCA } & 7\end{array}$

5 Comparison with lab measurements 9

6 Conclusions 12

\section{Introduction}

The Large Synoptic Survey Telescope (LSST) aims to explore the night sky in six optical bands ranging from 320 to $1100 \mathrm{~nm}$ [1]. LSST will have unprecedented precision to probe the nature of dark energy and dark matter, to take an inventory of the Solar System, to map the Milky Way, and to explore transient phenomena in the optical sky $[1,2]$. The LSST focal-plane array will have 21 Science Rafts each composed of nine $4 \mathrm{~K} \times 4 \mathrm{~K}$ CCDs with a pixel size of $10 \times 10 \mu \mathrm{m}$ [3]. The large field of view, 9.6 square degrees, and ability to read out all CCDs in only 2 seconds with a total exposure time of $15 \mathrm{sec}$, will allow LSST to image the entire accessible sky in only two to three days.

The fully depleted sensors are $100 \mu \mathrm{m}$ thick to improve their IR sensitivity. Figure 1 shows the total LSST throughput in the six bands. The $y$ band, which is most relevant to the studies described here, spans from about $900 \mathrm{~nm}$ to the silicon sensitivity cut-off at $1100 \mathrm{~nm}$. This band is most affected by fringing because this phenomenon occurs when light reflects off the back-surface of the CCD, so as the absorption depth becomes similar to the CCD's thickness (around $1000 \mathrm{~nm}$ ) the effect is greatly enhanced $[4,5]$. It should be noted that fringing can also occur in any filters which transmit wavelengths greater than $700 \mathrm{~nm}$.

Fringing patterns, discussed in more detail below, can exist in all images taken with longer wavelength light (though there are nights where an undetectable amount of fringing is possible; see below). Understanding the characteristics of the night-sky airglow that generates this longer 
wavelength light is important to understanding fringing in LSST images, and in particular to study its stability. A large relative variation in the amplitude of the emission lines causing fringing, coupled with sufficiently large thickness variations across a CCD, can produce a change in the fringe pattern itself, motivating the use of a more complex fringe removal algorithm in the LSST image processing pipeline. There have been several efforts to control and remove fringe patterns [69]. Specifically, [5] explores fringe removal via the use of a neon lamp as a way to approximate fringe patterns from the night sky, making use of observing time during the day to generate a fringe correction frame.

This experiment utilizes "MonoCam," a camera with a single LSST CCD read out by a commercial controller and equipped with an LSST $y 4$ filter. MonoCam collected data at the $1.3 \mathrm{~m}$ telescope at NOFS in Flagstaff, AZ in May 2016.

\section{$2 \mathrm{OH}$ airglow and fringing}

The dominant spectra from the night sky with respect to fringing occur from hydroxyl $(\mathrm{OH})$ emission lines $[10,11]$. As the night progresses, it is expected that these emission lines shift in their relative intensities [12] and, as a result, cause the fringe patterns observed in the LSST CCDs to potentially change as well. It is possible for nights to contain negligible $\mathrm{OH}$ emission (and therefore have little to no fringing). Shown in figure 2 are the relative theoretical $\mathrm{OH}$ emission line intensities in the range $900 \mathrm{~nm}$ to $1100 \mathrm{~nm}$, the same range that the $y 4$ filter roughly covers (see figure 1). Emission line intensities are from [13].

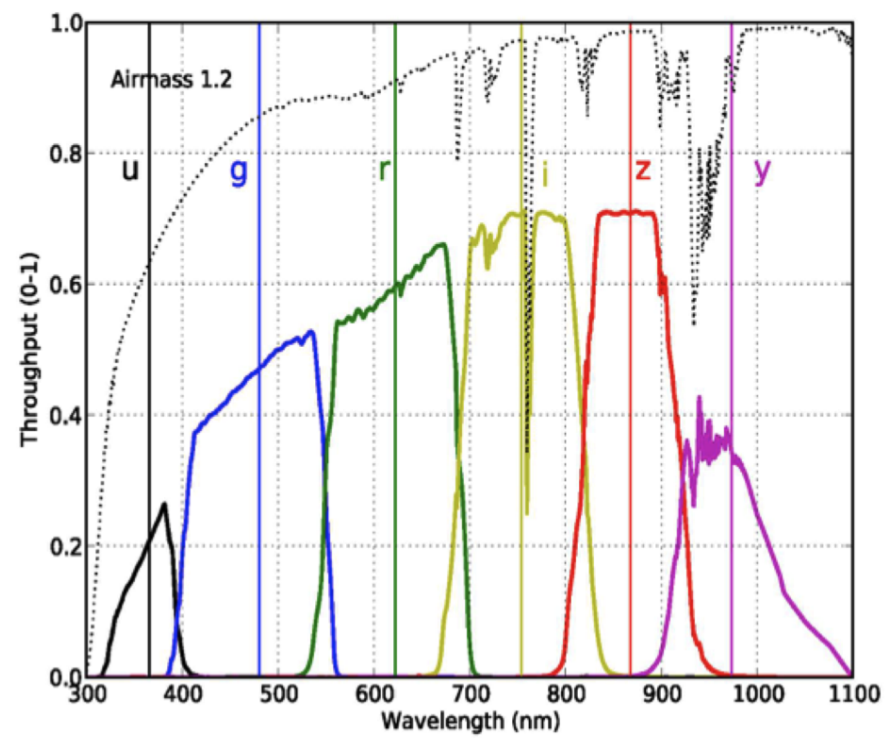

Figure 1. LSST filter bands [14]. All images analyzed in this paper were taken with the Y4 filter ("y" filter shown in pink in this image). The dotted line represents the throughput or transmission of the atmosphere at a given wavelength.

Fringing occurs because long wavelength light reflects back inside a CCD and creates interference patterns due to the varying sensor thickness $[5,15]$ The fringes are noticeable on all images recorded for these studies, see figure 3 which shows images taken throughout the night. These 


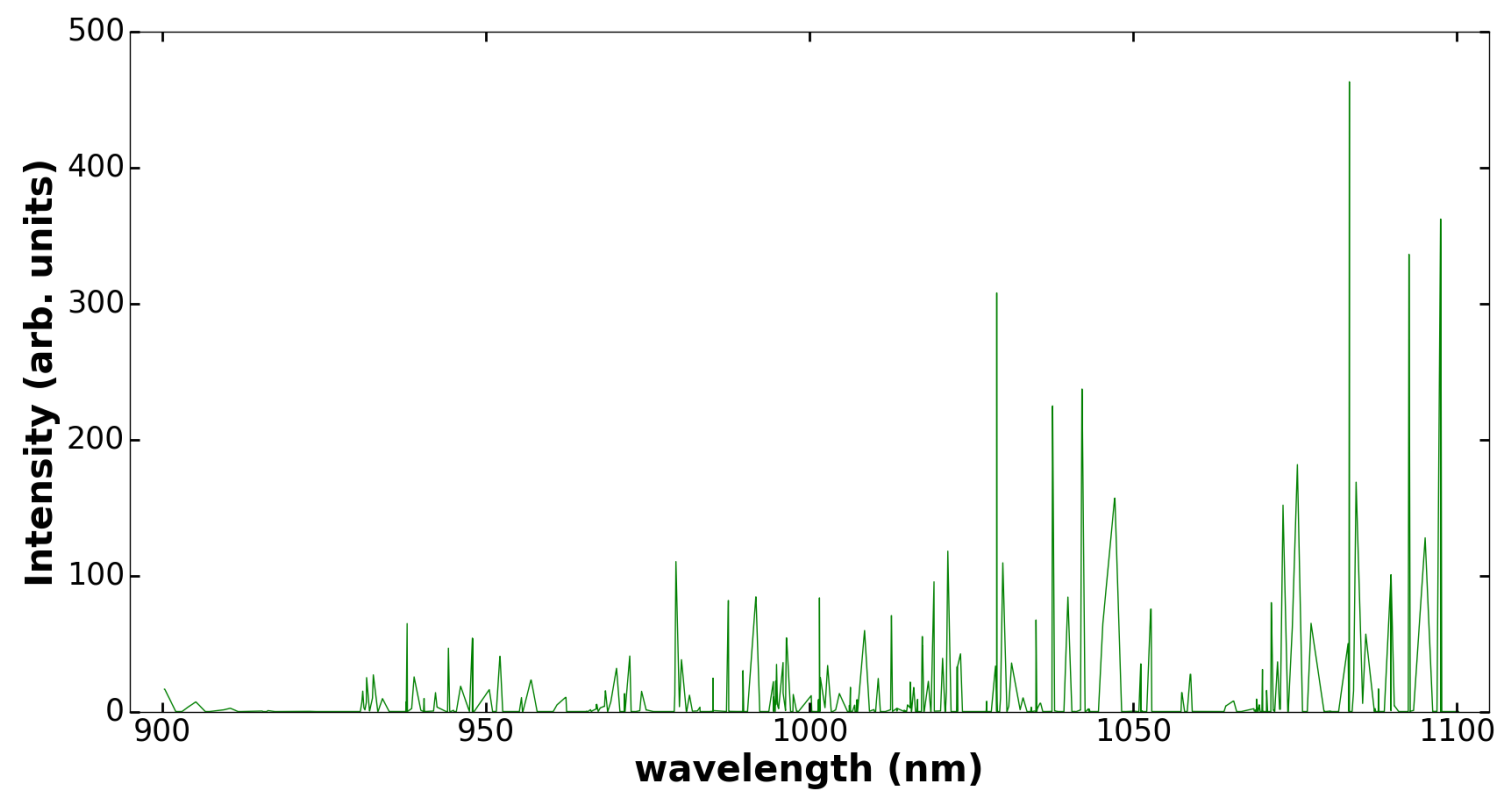

Figure 2. Theoretical $\mathrm{OH}$ line emission intensities from [13].

images are discussed in more detail in the next section. We can relate fringe patterns to the varying thickness of a CCD using the following equation [15]:

$$
\Delta x=\frac{\delta \lambda \cos (\theta)}{4 \pi n_{\mathrm{Si}}}
$$

the variable $\Delta x$ represents the variation in thickness $(\mu \mathrm{m}), \delta$ the phase difference of the fringe pattern, $\lambda$ the wavelength of the incident light (in $\mu \mathrm{m}$ ), $\theta$ the angle from normal in the silicon, and $n_{\mathrm{Si}}$ the index of refraction of silicon. As an example, if we assume that fringes are created using monochromatic light of wavelength $1 \mu \mathrm{m}$, and light is incident normal to the surface of the CCD, then the thickness variation required to see one fringe $(\delta=2 \pi)$ would be $0.14 \mu \mathrm{m}$. It should be noted that reflection inside the CCD can occur at multiple layers and with multiple wavelengths of light, causing several fringe patterns to superimpose in the images that are recorded.

\section{MonoCam}

MonoCam employs a single LSST prototype sensor, CCD250 113-03, produced by e2v [16]. The sensor is divided up into two rows of eight $512 \times 2002$ amplifier regions stacked side-by-side for a total size of $4096 \times 4004$ pixels, and is read out by a commercial Reflex controller [17] using RTS-2 software [18]. MonoCam was placed in a cryostat cooled with liquid nitrogen to keep the $\mathrm{CCD}$ back biased to $-70 \mathrm{~V}$ at a temperature of $-120 \mathrm{C}$ under vacuum of about $10^{-6}$ torr. A Keithley source meter 2410 was used to provide the back side bias for the sensor. All equipment including the CCD cryostat was used for LSST CCD R\&D at BNL between 2008-2015, and is described in more detail elsewhere [19]. 


\subsection{Telescope}

The images shown in figure 3 were taken at the U.S. Naval Observatory located at Flagstaff, Arizona on the night of May 11 and the morning of May 12, 2016 using its $1.3 \mathrm{~m}$ telescope. All images studied in this paper came from the blank field CABlank5 at RA 162433 and DEC +55 4359 and were observed with the $y 4$ filter.

The telescope used was a reflector with an image scale of $39.6 \mathrm{arcsec} / \mathrm{mm}$ and an overall focal ratio of $f / 4.0$. The diameter of the primary mirror is $1.3 \mathrm{~m}$ with a focal ratio of $f / 2.4$ and has a concave hyperboloid figure. The secondary mirror has a diameter of $0.61 \mathrm{~m}$ and a convex hyperboloid figure. The MonoCam field of view at this telescope is $0.44 \times 0.44$ square degrees [20].

\subsection{Data acquisition}

Images were taken every hour throughout the night, starting at $8: 25 \mathrm{pm}$. At each hour, ten backto-back 60-second exposures were taken, though exposures for images recorded between 2:41am and 2:59am were 120 seconds long rather than 60 seconds. In addition, four out of the ten images recorded starting at 1:43am failed to pass through the data reduction process, so there are only six images available to study during this ten minute period. Lastly, throughout this paper we will refer to each set of hourly images (or average of the images for each hour) by the time that images began to be taken at that hour.

\subsection{Data reduction}

Images that were analyzed in this paper were first run through LSST Data Management software [21, 22]. This software was used to generate "postISRCCD" images that utilized flat field correction and bias subtraction to create the images shown in figure 3. Flat field correction helps to account for unwanted static features on the images, as well as differences in amplifier gain. Bias subtraction aids in removing amplifier offsets on a pixel-by-pixel basis.

\subsection{Observed patterns}

The patterns shown in figure 3 represent the average of these hourly ten-image segments taken throughout the night. From this figure it is apparent that the fringe pattern does not fluctuate much (see the following section where changes in the patterns are examined more closely). However, sky background levels which are displayed above each image in figure 3 change from about 230 analog-to-digital units (ADU) at 8:25pm to 90ADU at 1:43am. Note that the high sky background in the 2:41am image is due to the longer exposure time (120 seconds instead of 60 seconds per image at this hour). The telescope also observed different star fields during the course of the night, and would periodically return to observe this blank field, so there are minor changes in the star positions in each image due to the telescope's pointing variation. It should also be noted that we are only looking at fringe patterns across one night; our analysis could be improved with the addition of other nights.

Note also the faint secondary fringe pattern that appears to radiate away from the center of each image. This secondary pattern could arise due to interference at another layer (or layers) inside the CCD. Lastly, note a line originating in the top left corner of each image which separates fringing regions with a slight phase shift, a so called "spider leg". This effect is caused by a small step in the silicon thickness due to the grinding process to achieve the nominal sensor thickness [16]. 


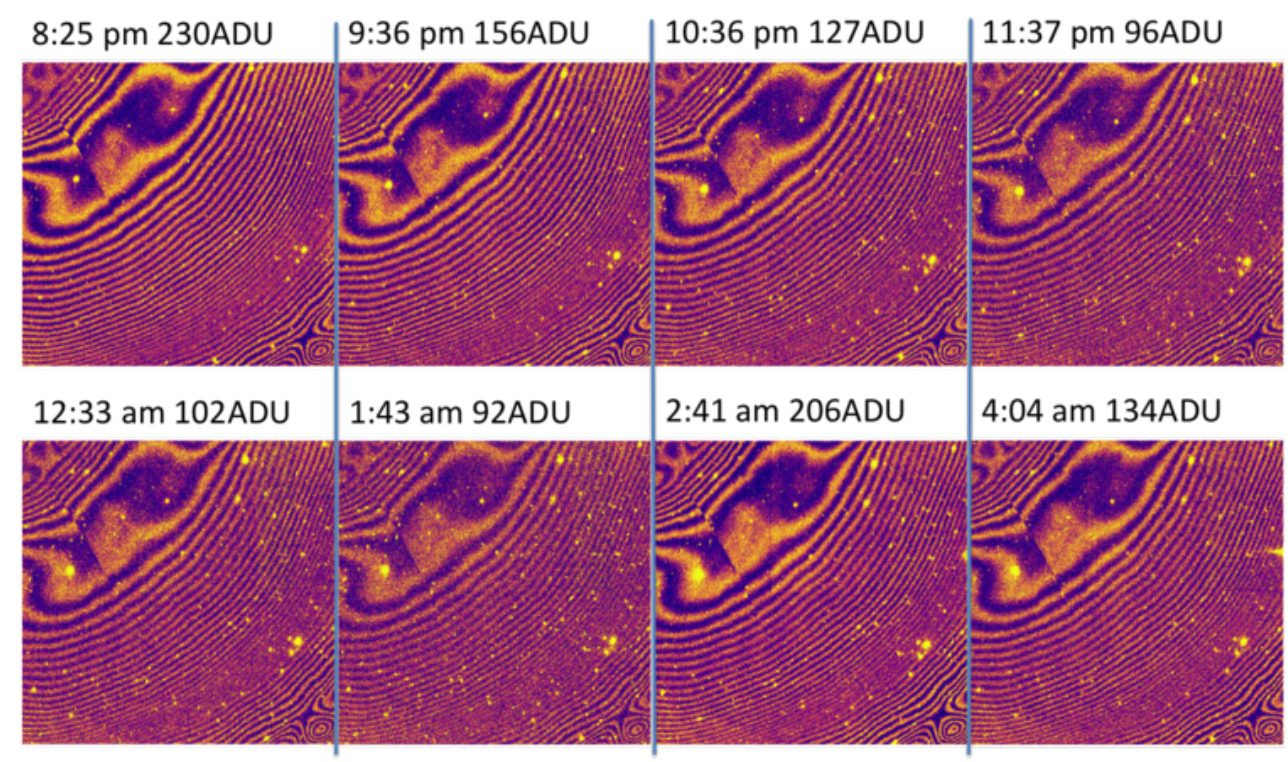

Figure 3. Fringe patterns observed throughout the night. The time listed above each image gives the start of the ten-minute period over which the images were taken. The resultant image is an average of each of these ten images. The value next to the time shows the median pixel value for the particular hour displayed, giving an estimate of the sky background level. The scaling in each image changes in order to highlight the structure of the fringe patterns.

\section{Data Analysis}

\subsection{Subtraction by midnight pattern}

From the hours observed during the night, we arbitrarily chose and subtracted the 12:33am (midnight) image from each image shown in figure 3 . Before the subtraction was performed, the images were normalized, i.e. the midnight image was first divided by its median value and then multiplied by the median value of the image it was being subtracted from. This process showed that relative fringe amplitudes observed close to the midnight hour, for example at $11: 37 \mathrm{pm}$ and $1: 43 \mathrm{pm}$, were reduced from about $2 \%$ in the original images to less than $0.5 \%$ in the subtracted images. At times further from the midnight hour, the subtraction becomes less effective; fringe amplitudes at times like $8: 25 \mathrm{pm}$ and $2: 41 \mathrm{am}$ are reduced from about $2-3 \%$ of the image median value to $1-1.5 \%$ of the median. An interesting point to note here is the fact that dark spots in images taken before midnight in figure 4 roughly correspond to the bright spots in all images taken after midnight. This implies that there could be a subtle phase shift or change in the amplitude of the fringe pattern as it progresses throughout the night; see figure 5 for a closer look at how the phase of the slice plots changes.

\subsection{Principal Component Analysis}

Principal Component Analysis (PCA) is performed by finding the eigenvectors of the covariance matrix formed by the dataset in question [24, 25]. These principal components (eigenvectors) are then ranked in descending order, based on their associated eigenvalue, on the assumption that the dimensions of the data with the highest variance represent the most interesting aspects of the data 


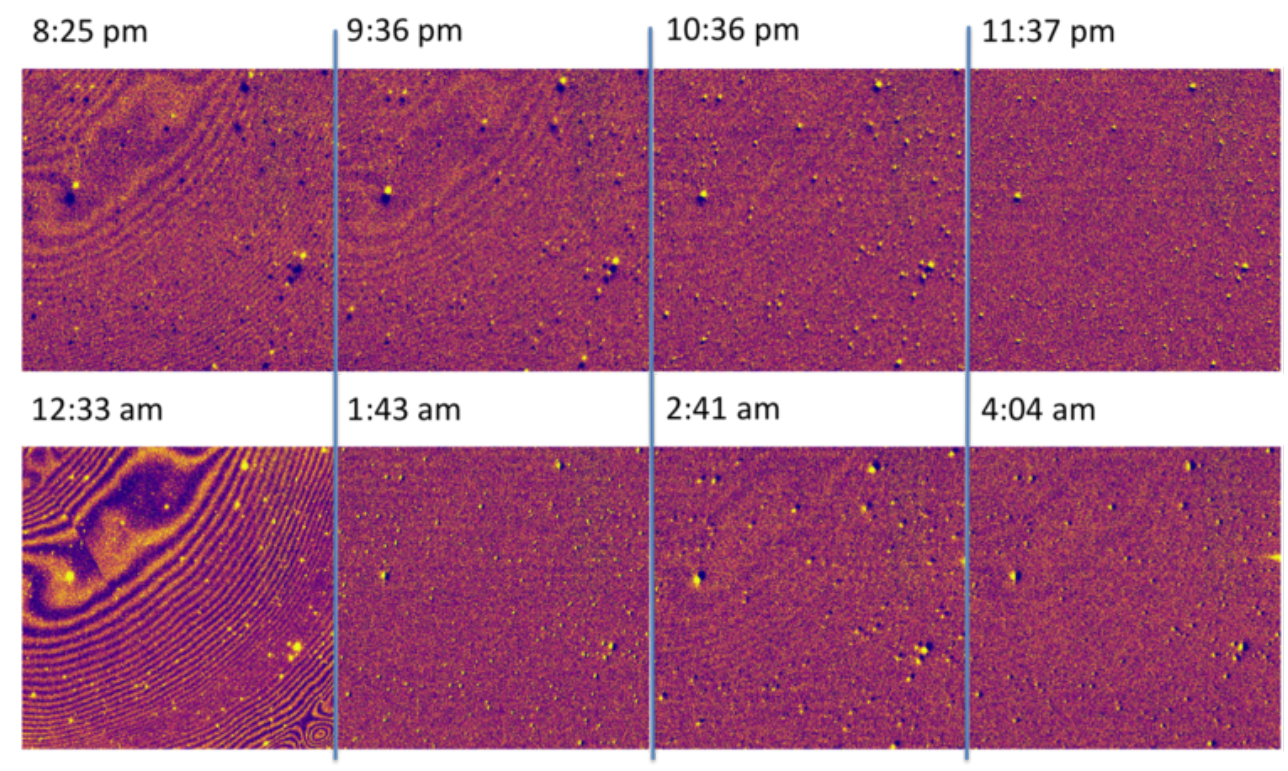

Figure 4. Fringe patterns observed throughout the night that have had the scaled midnight pattern subtracted from them. For reference, the midnight pattern is included in the bottom left corner.
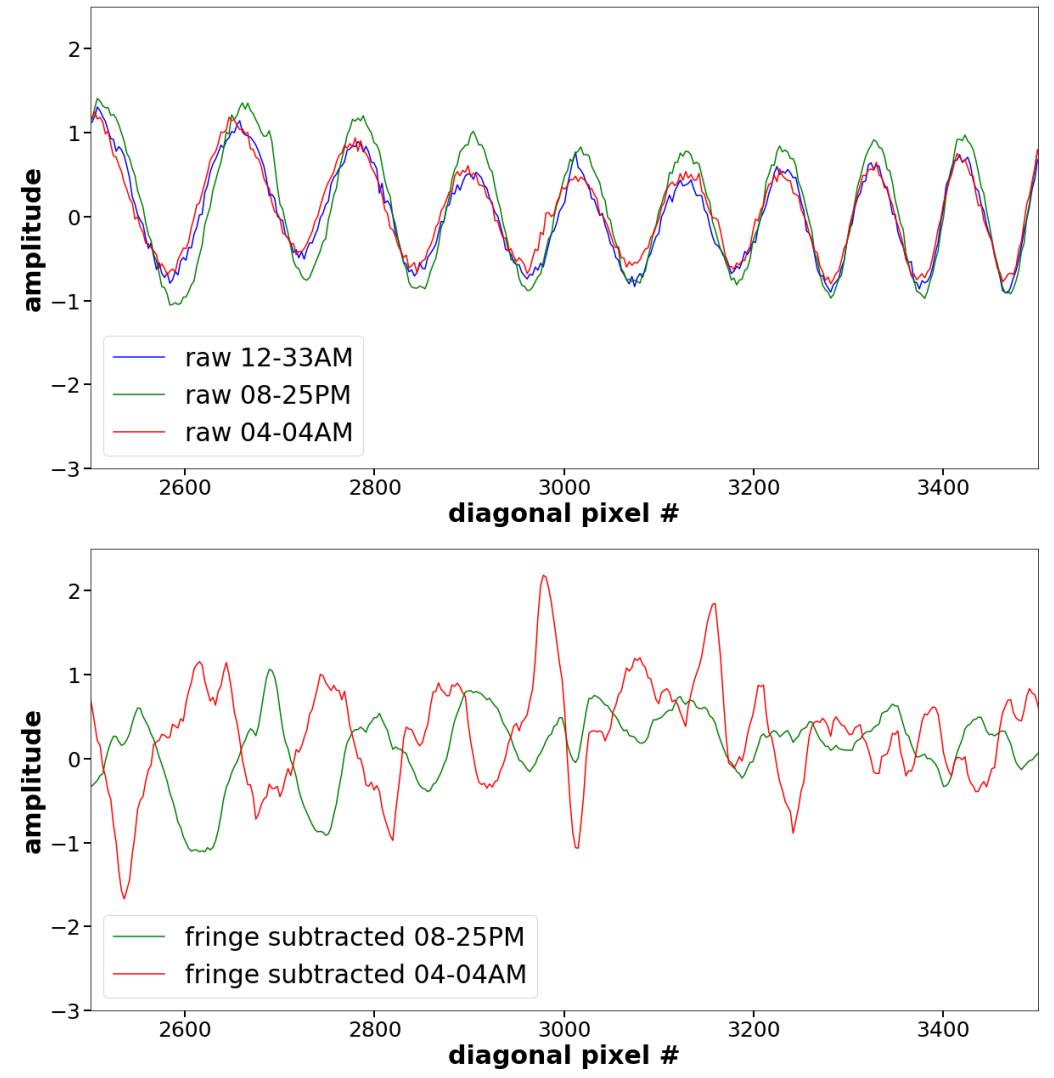

Figure 5. Top plot: A section of the raw slice plots for 8:25pm, 12:33am, and 4:04am. We can see that the three lines are slightly out of phase. Bottom plot: Slice plots of the 8:25pm and 4:04am images with the midnight pattern subtracted from them. All slice plots were run through smoothing and median-centering software [23] and then divided by their interquartile range. 
being analyzed. In a sense, PCA tries to capture what "changes most" in a data set and allows one to ignore aspects of the data that are just noise. After the principal components are found, we can reconstruct our data (or approximate new observations) via the following formula [26]:

$$
\vec{y}=\vec{\mu}+\sum_{k} c_{k} \vec{\phi}_{k}
$$

where $\vec{y}$ is the observed data or new data to be approximated, $\vec{\mu}$ is the average of the data set used to perform the PCA, and $c_{k}$ is the $k^{\text {th }}$ coefficient (discussed more below) multiplied by its associated eigenvector, $\vec{\phi}_{k}$. Because the eigenvectors are ranked in descending order, $\vec{y}$ can usually be approximated using only the first few values in the sum. As stated above, $\vec{\phi}_{k}$ is the $k^{\text {th }}$ eigenvector found from the covariance matrix of the data, and $c_{k}$ is found by projecting a data observation onto the set of eigenvectors [26]:

$$
(\vec{y}-\vec{\mu}) \cdot \vec{\phi}_{k}=c_{k}
$$

Note that the values of $c_{k}$ are different for each observation $\vec{y}$.

The analysis in this paper uses scikit-learn's PCA code [27] that utilizes the Linear Algebra Package (LAPACK) implementation of the full Singular Value Decomposition (SVD) to compute the principal components and weights $c_{k}$. This process is not the same as the standard PCA described above, but produces equivalent results.

\subsection{Slice plot PCA}

Points across the upper left to bottom right diagonal of the images shown in figure 3 were used in the following PCA. Before taking the slice plot, we used software [23] to median-subtract each image. This same software was then used to smooth out each slice plot using a transverse Gaussian smoothing procedure that reduced noise and smoothed out stars along the slice line. Lastly, each data hour was treated like a vector and normalized to unit-length using scikit [27] software.

Figure 6 shows the principal components of our data set, which consisted of the slice plots derived from the eight observations shown in figure 3 . We can see that the mean and first component contain most of the interesting information in our data; components two through eight appear to be minor contributions from noise and stars. It should be noted that all components (and the mean) are centered around zero, but in this figure they are given an offset in order to display all eigenvectors in one plot. Figure 8 shows how the relative weights $c_{k}$ change as the night progresses. This plot shows clear evidence that there are minor shifts in the fringe pattern throughout the night. Component 1's weights have the largest change in value throughout the night, from +0.31 at $8: 25 \mathrm{pm}$, to approximately -0.17 at $2: 41 \mathrm{am}$, reflecting the fact that this component mostly accounts for changes in the structure of the hourly fringes, while other components account more for changes in the noise. Furthermore, figure 7 shows a closeup of the mean and first principal component plotted over each other. We can see the change in phase between the two lines that offers more evidence for a phase shift occurring in the nighttime fringe patterns as it changes throughout the night that we observed.

In the future, we could improve our analysis by performing PCA on the entire image for each hour in the MonoCam data. An obstacle that must first be overcome is the fact that stars litter each image, and, if left alone, will dominate the PCA. Smarter techniques, like those described in [26], could be used to deal with this and further improve the analysis. 


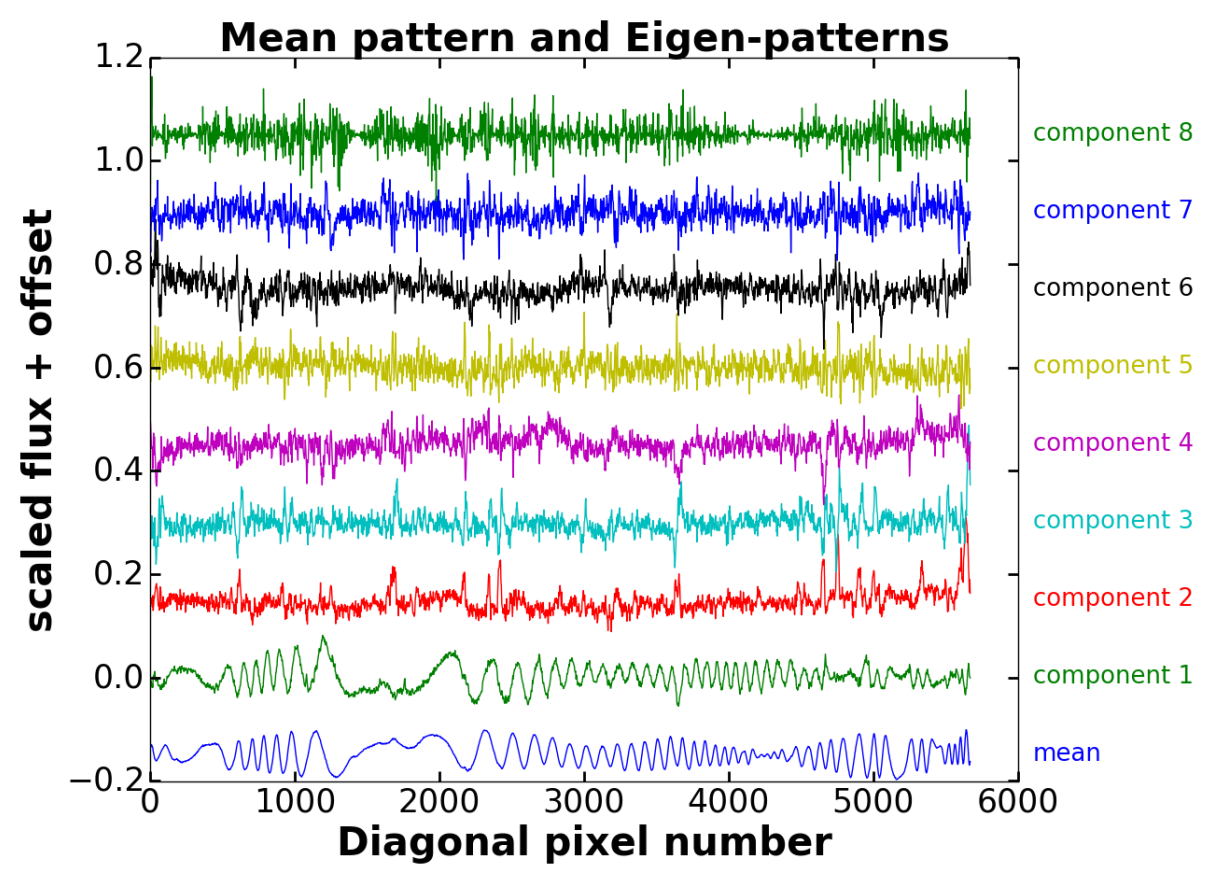

Figure 6. Mean and eigen-patterns (principal components) computed by performing PCA on the slice plots from the MonoCam data. Components are shown in ascending order in this plot; the component with the highest variance (component 1 ) is shown at the bottom, the component with the second highest variance (component 2) is shown above, and so on.

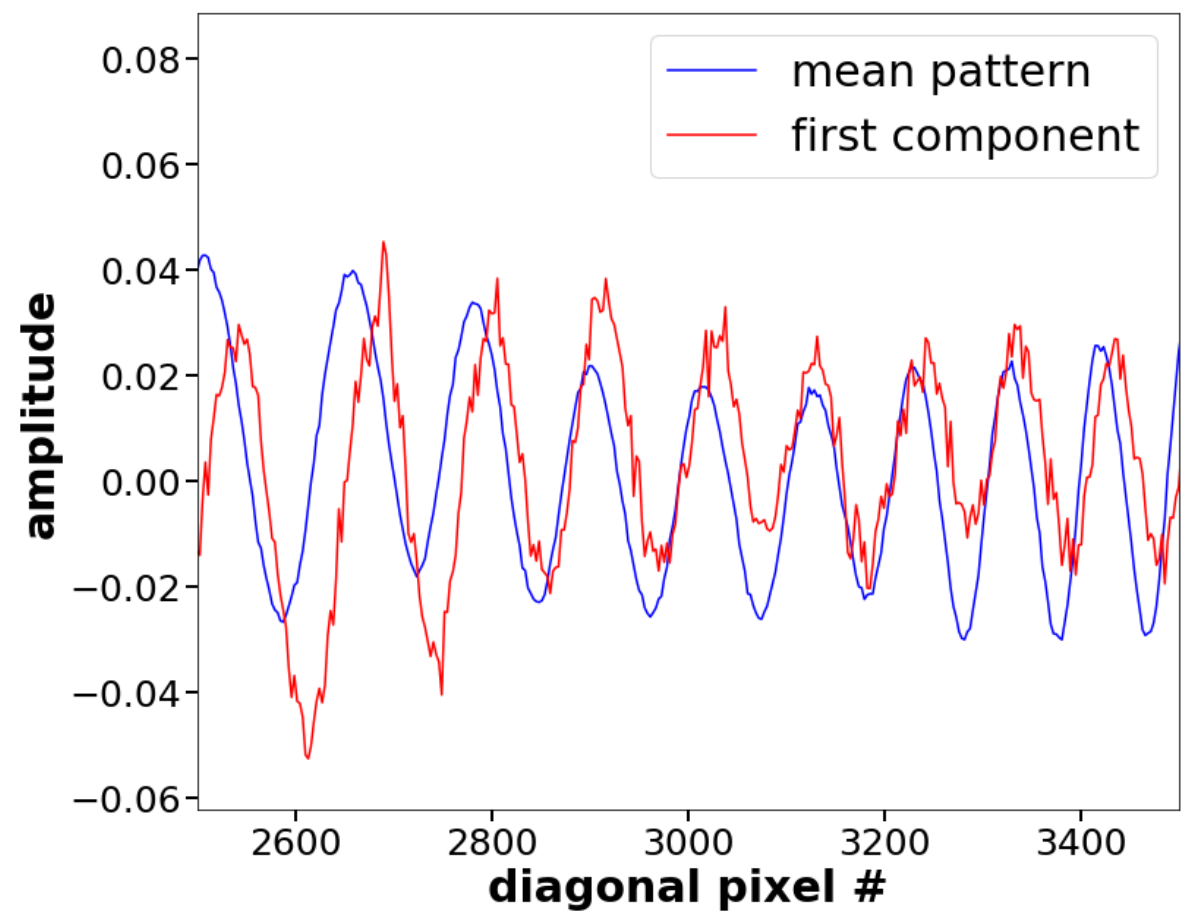

Figure 7. Mean and pca component one plotted together in the range 2500-3500. We can see that there is a slight phase shift between the two lines. 


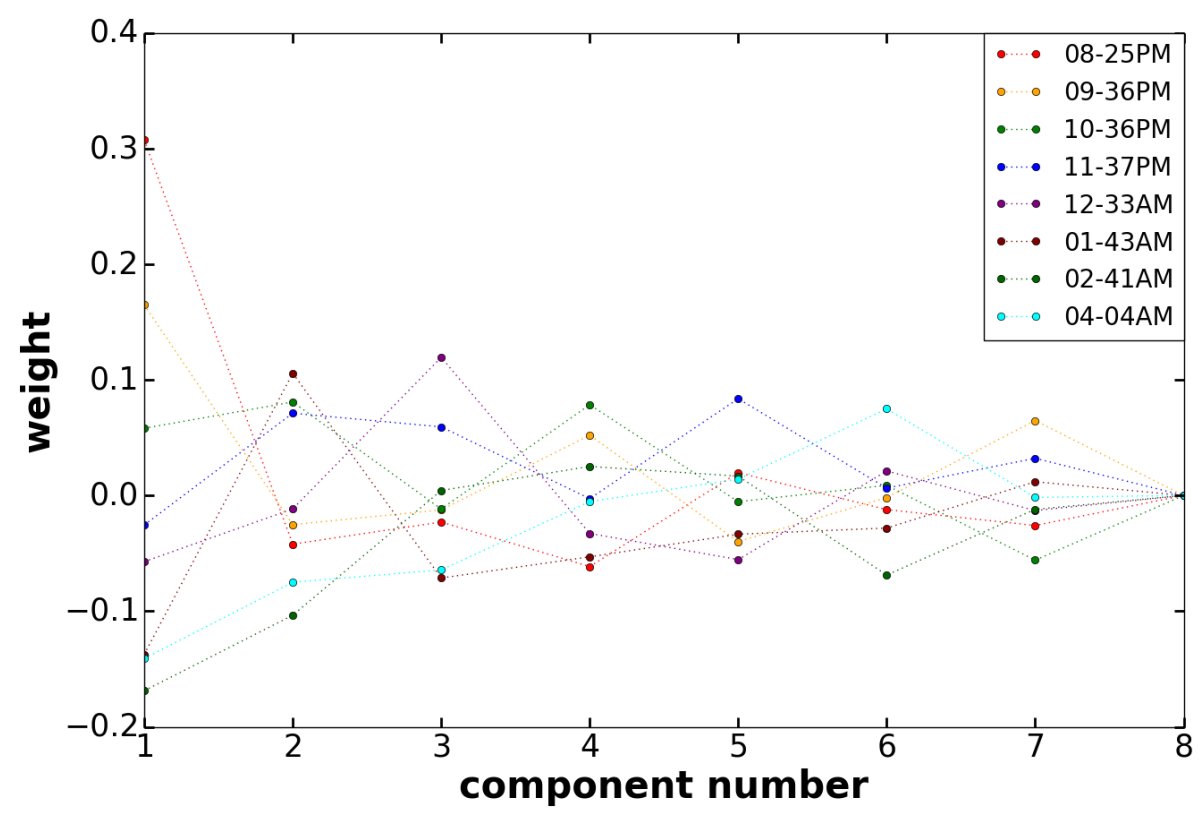

Figure 8. Weights $c_{k}$ plotted on the $x$-axis versus their values on the $y$-axis for each hour. Note how the weight associated with the first component changes the most throughout the night.

\section{Comparison with lab measurements}

Lab flats acquired with the same CCD as used in MonoCam were taken in increments of $10 \mathrm{~nm}$ over the range $900 \mathrm{~nm}$ to $1100 \mathrm{~nm}$. This range closely approximates the range of the LSST $y 4$ filter shown in figure 1. We attempt to fit a superposition of these lab flats to the observed MonoCam data at midnight (given by the vector $\vec{y}_{\text {mid }}$ ) using the following formula:

$$
\vec{y}_{\text {mid }}=\sum_{n=0}^{20} \alpha_{n} \vec{\psi}_{n}
$$

where index $n$ is related to the wavelength in question: $n=0$ corresponds to the $900 \mathrm{~nm}$ lab flat, $n=1$ the $910 \mathrm{~nm}$ lab flat, and so on. $\vec{\psi}_{n}$ represents the $n^{\text {th }}$ lab flat and $\alpha_{n}$ represents the optimized weight multiplied by the lab flat to reconstruct $\vec{y}$. The values of $\alpha_{n}$ were computed using code written in Python which utilizes SciPy optimization functions [28]. As before, we only use the slice plots of the lab flats extending from the top left corner of each image to the bottom right corner, and fit these plots to the MonoCam midnight slice plot. Before slice plots of the lab data are thrown into the optimization procedure, they are median subtracted and smoothed using the same software [23] that was used to smooth the slice plots in our PCA. After that, they are also divided by their interquartile range, and multiplied by the respective $y 4$ filter throughput value, as shown in figure 1 .

The top plot in figure 9 shows the line generated using equation (5.1) and optimized parameters $\alpha_{n}$ that best fit the midnight slice plot. Figure 10 displays the values of $\alpha_{n}$ found through the optimization procedure. Based on this plot we could conclude that at midnight the emission lines at $1000 \mathrm{~nm}$ and $1030 \mathrm{~nm}$ dominate the spectrum based on the two highest weights $\alpha_{n}$, shown in figure 10 .

It can also be shown that the node in the MonoCam slice plot around diagonal pixel number 4300 can be recreated in the bottom plot of figure 9 using just a superposition of two lab flat slice 

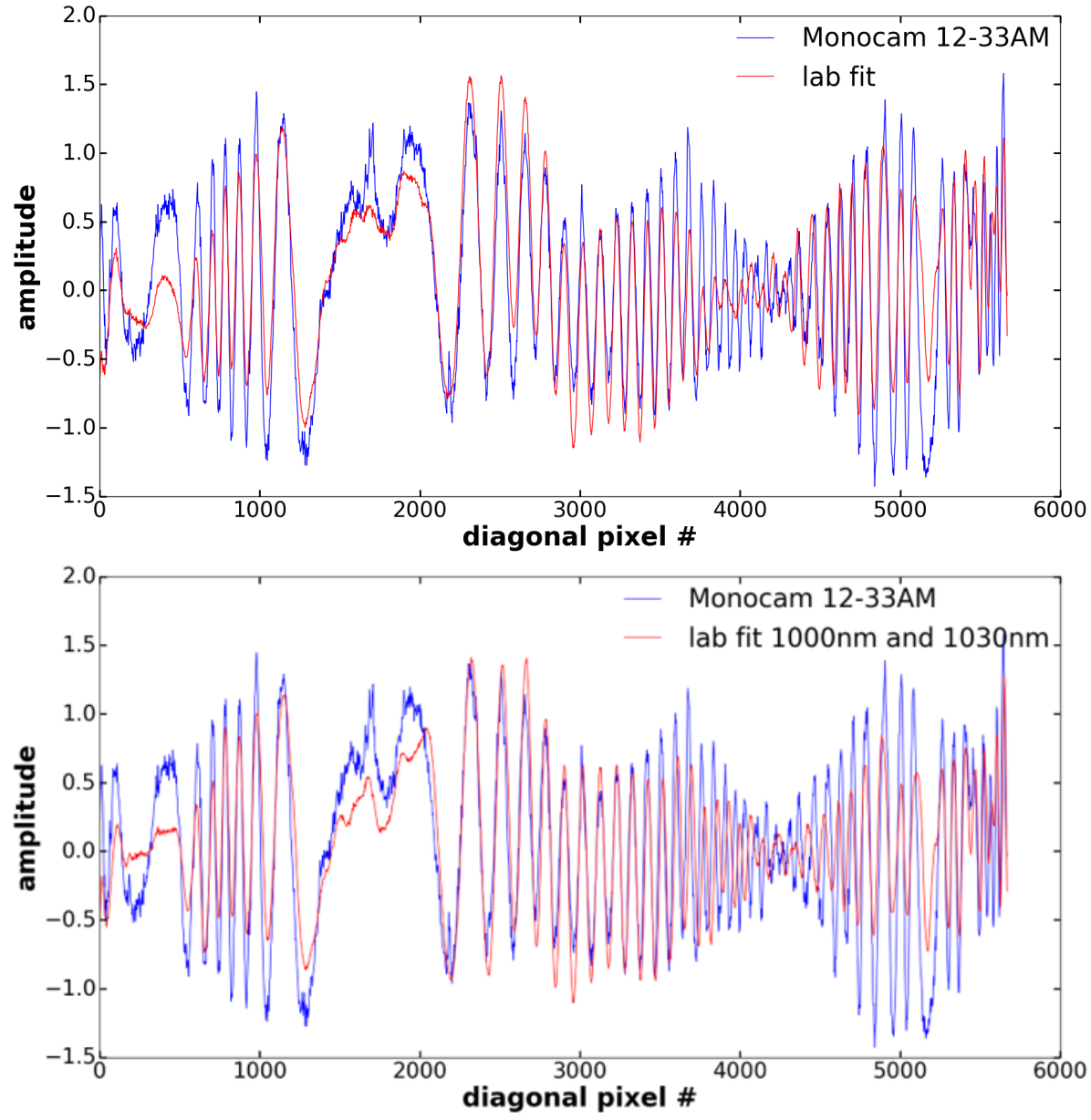

Figure 9. Top plot: Lab fit using optimized parameters $\alpha_{n}$ from equation (5.1) shown in red, plotted against the observed midnight pattern. Bottom plot: Lab fit to midnight hour data using only two lab flats at wavelengths $1000 \mathrm{~nm}$ and $1030 \mathrm{~nm}$.

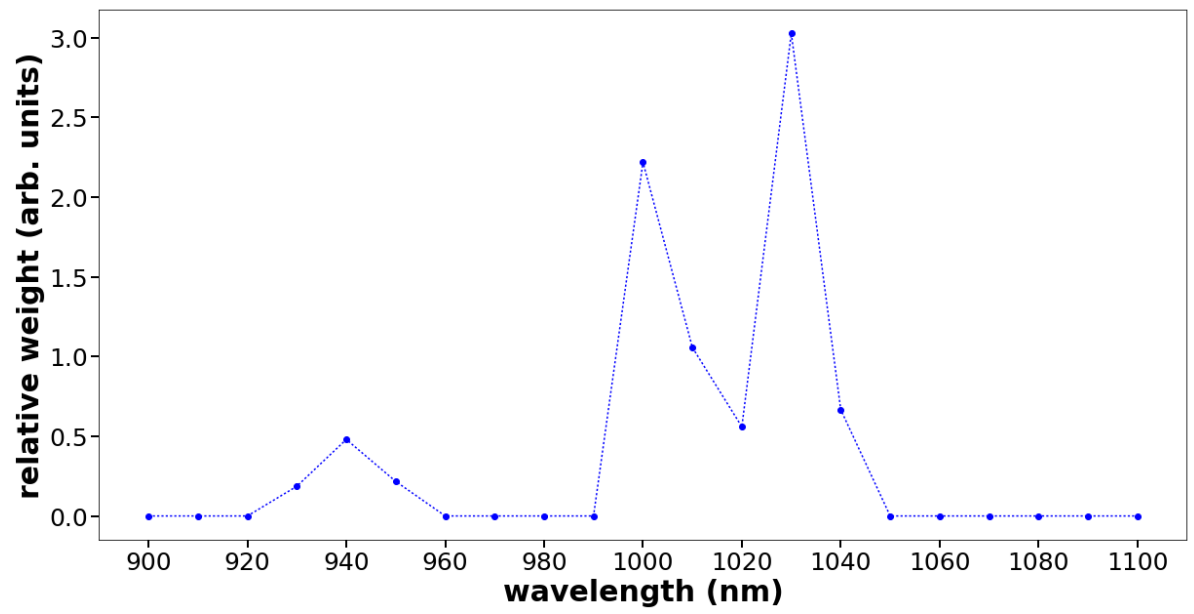

Figure 10. Coefficients $\alpha_{n}$ plotted on the $x$-axis versus their weights plotted on the $y$-axis used in equation (5.1) to create figure 9. If the results from this fit are accurate, then at midnight emission lines around $1000 \mathrm{~nm}$ and $1030 \mathrm{~nm}$ dominate the night sky airglow. 

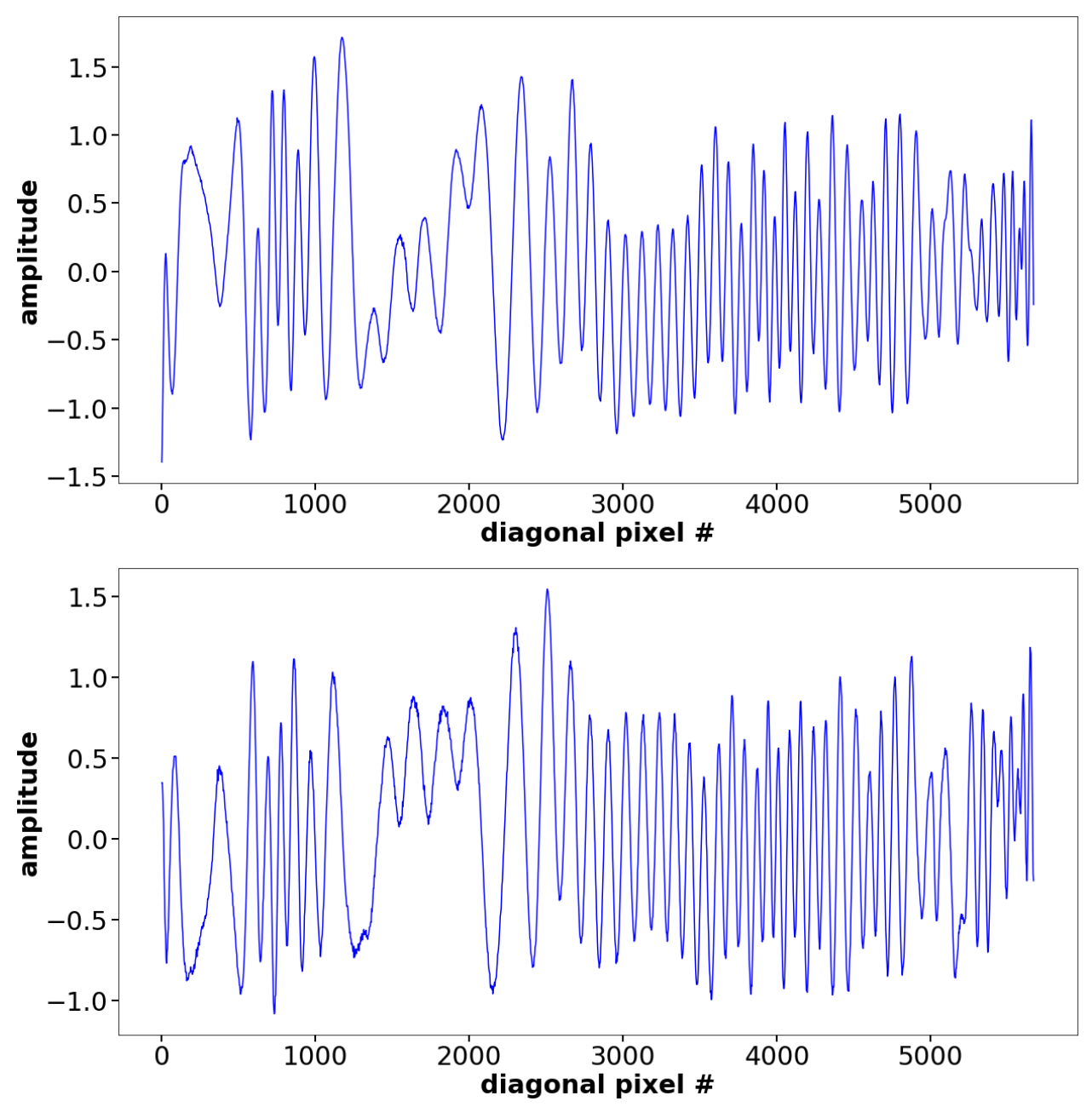

Figure 11. Top plot: Slice plot of a monochromatic lab flat taken at $1000 \mathrm{~nm}$. Bottom plot: Slice plot of a monochromatic lab flat taken at $1030 \mathrm{~nm}$.

plots at $1000 \mathrm{~nm}$ and $1030 \mathrm{~nm}$. These two lab flat slice plots are shown in figure 11. Recall that a sum of two sinusoids can create beat patterns, and this procedure shows that we could recreate this beat with just two lab flats. In addition, this finding appears to be consistent with the two highest points in figure 10; the enveloping structure of the observed data can be approximated with the lab flats associated with the highest weights in figure 10.

It is apparent that the fitted line shown in figure 9 does not appear to fully describe behavior of the midnight slice plot. There are several factors that could account for discrepancies in the lab fit. For example, we only have twenty-one lab flats to use, taken with a step of $10 \mathrm{~nm}$; if we had more (maybe one flat per nanometer in the range $900 \mathrm{~nm}-1100 \mathrm{~nm}$ ) it is possible that the fitting procedure would produce more accurate results. In addition, the discrepancy may arise from the $3 \mathrm{~nm}$ bandwidth of the monochromatic light source used to generate the lab flats, which may have caused a difference in shapes of the fringes. Yet another reason could be differences in the angular distribution of the incident light; the lab flats were taken with practically parallel illumination from an integrating sphere, whereas for MonoCam the light was coming from a telescope with f/4.0. 


\section{Conclusions}

We observed fringe patterns in images taken with MonoCam, a camera based on a single LSST CCD, and employing an LSST $y 4$ filter and the $1.3 \mathrm{~m}$ telescope at NOFS at Flagstaff AZ. The major fringe pattern does not appear to change significantly over the course of the night. However, two different techniques described above revealed that there are subtle changes in the shape of the fringes occurring throughout the night. The phase of the fringes we measured change with time as shown directly in figure 5 and through our Principal Component Analysis. However, more work is needed to understand in more detail the subtle changes these fringe patterns undergo throughout the night. Comparison of the sky data and the lab flats taken with the same sensor suggests that emission lines around $1000 \mathrm{~nm}$ and $1030 \mathrm{~nm}$ dominate the night sky spectrum, though the best fit has discrepancies with the observed sky data.

\section{Acknowledgments}

We would like to thank Kirk Gilmore for providing the filter throughput plot shown in figure 1, in addition to his assistance throughout this project. We would also like to thank David Kirkby, whose code was used to produce several of the plots shown. Lastly, we thank Petr Kubanek, Woodrow Gilbertson, David Monet and the Naval Observatory telescope personnel for their help with the camera setup and operation, and data taking. This project was supported in part by the U.S. Department of Energy, Office of Science, Office of Workforce Development for Teachers and Scientists (WDTS) under the Science Undergraduate Laboratory Internships Program (SULI). This paper makes use of software developed for the Large Synoptic Survey Telescope. We thank the LSST Project for making their code available as free software at http://dm.lsst.org.

\section{References}

[1] LSST SCIENCE collaboration, LSST Science Book, Version 2.0, arXiv:0912.0201.

[2] M. Jurić et al., LSST: from Science Drivers to Reference Design and Anticipated Data Products, arXiv:0805.2366.

[3] LSST technical details, https://www.lsst.org/about/camera/features.

[4] F.W. High, C.W. Stubbs, B. Stalder, D.K. Gilmore and J.L. Tonry, Sky Variability in the y Band at the LSST Site, Publ. Astron. Soc. Pac. 122 (2010) 722.

[5] S.B. Howell, Fringe Science: Defringing CCD Images with Neon Lamp Flat Fields, Publ. Astron. Soc. Pac. 124 (2012) 263 [arXiv: 1201.2336].

[6] D. Tucker et al., The sloan digital sky survey monitor telescope pipeline, Astron. Nachr. 327 (2006) 821.

[7] D.G. Gilbank, M.D. Gladders, H.K.C. Yee and B.C. Hsieh, The Red-sequence Cluster Survey-2 (RCS-2): survey details and photometric catalog construction, Astron. J. 141 (2011) 94 [arXiv: 1012.3470].

[8] M. Irwin and J. Lewis, \{INT\} \{WFS\} pipeline processing, New Astron. Rev. 45 (2001) 105.

[9] C.R. Gelino, M.S. Marley, J.A. Holtzman, A.S. Ackerman and K. Lodders, L-dwarf variability: i-band observations, Astrophys. J. 577 (2002) 433 [astro-ph/0205305]. 
[10] A.B. Meinel, OH emission bands in the spectrum of the night sky I, Astrophys. J. 111 (1950) 555.

[11] A.B. Meinel, OH emission bands in the spectrum of the night sky II, Astrophys. J. 112 (1950) 120.

[12] D.R. Marsh, A.K. Smith, M.G. Mlynczak and J.M. Russell, Saber observations of the OH meinel airglow variability near the mesopause, J. Geophys. Res. 111 (2006) A10S05.

[13] P. Rousselot, C. Lidman, J.-G. Cuby, G. Moreels and G. Monnet, Night-sky spectral atlas of OH emission lines in the near-infrared, Astron. Astrophys. 354 (2000) 1134.

[14] K. Gilmore, personal communication, November 2016.

[15] J.R. Janesick, Scientific Charge-Coupled Devices, SPIE, Bellingham, Washington, U.S.A. (2001).

[16] P.R. Jorden, D. Jordan, P.A. Jerram, J. Pratlong and I. Swindells, e2v new ccd and cmos technology developments for astronomical sensors, Proc. SPIE 9154 (2014) 91540M.

[17] Reflex: a high performance modular CCD controller, Semiconductor Technology Associates, Inc., 27122 Paseo Espada, Ste 1004, San Juan Capistrano, CA 92675, 2011, http://www.sta-inc.net/wp-content/uploads/2011/04/Reflex.pdf.

[18] P. Kubánek et al., RTS2 - remote telescope system, $2^{\text {nd }}$ Version, AIP Conf. Proc. 727 (2004) 753.

[19] P. O’Connor, J. Frank, J.C. Geary, D.K. Gilmore, I. Kotov, V. Radeka, P. Takacs and J.A. Tyson, Characterization of prototype lsst ccds, Proc. SPIE 7021 (2008) 702106.

[20] The 1.3-m Reflector, http://www.nofs.navy.mil/.

[21] M. Jurić et al., The LSST data management system, 2015, arXiv: 1512 . 07914.

[22] T. Axelrod, J. Kantor, R.H. Lupton and F. Pierfederici, An open source application framework for astronomical imaging pipelines, Proc. SPIE 7740 (2010) 774015.

[23] D. Kirkby, software available at https://github.com/dkirkby/AstroCCD.

[24] I.T. Jolliffe, Principal component analysis, second edition, Springer series in statistics, Springer-Verlag, New York, New York, 2002.

[25] J. Shlens, A tutorial on principal component analysis, 2005, http://www.cs.cmu.edu/ elaw/papers/pca.pdf.

[26] S. Bailey, Principal Component Analysis with Noisy and/or Missing Data, Publ. Astron. Soc. Pac. 124 (2012) 1015 [arXiv: 1208.4122].

[27] F. Pedregosa, G. Varoquaux, A. Gramfort, V. Michel, B. Thirion, O. Grisel et al., Scikit-learn: Machine learning in Python, J. Mach. Learn. Res. 12 (2011) 2825.

[28] E. Jones, T. Oliphant, P. Peterson et al., SciPy: Open source scientific tools for Python, 2001, http://www.scipy.org/. 